\title{
Histone modification profiles are predictive for tissue/cell-type specific expression of both protein-coding and microRNA genes
}

\author{
Zhihua Zhang and Michael Q Zhang*
}

\begin{abstract}
Background: Gene expression is regulated at both the DNA sequence level and through modification of chromatin. However, the effect of chromatin on tissue/cell-type specific gene regulation (TCSR) is largely unknown. In this paper, we present a method to elucidate the relationship between histone modification/variation (HMV) and TCSR.

Results: A classifier for differentiating CD4+ T cell-specific genes from housekeeping genes using HMV data was built. We found HMV in both promoter and gene body regions to be predictive of genes which are targets of TCSR. For example, the histone modification types H3K4me3 and H3K27ac were identified as the most predictive for $\mathrm{CpG}$-related promoters, whereas H3K4me3 and H3K79me3 were the most predictive for nonCpG-related promoters. However, genes targeted by TCSR can be predicted using other type of HMVs as well. Such redundancy implies that multiple type of underlying regulatory elements, such as enhancers or intragenic alternative promoters, which can regulate gene expression in a tissue/cell-type specific fashion, may be marked by the HMVs. Finally, we show that the predictive power of HMV for TCSR is not limited to protein-coding genes in CD4+ T cells, as we successfully predicted TCSR targeted genes in muscle cells, as well as microRNA genes with expression specific to CD4+ $T$ cells, by the same classifier which was trained on HMV data of protein-coding genes in CD4+ $T$ cells.

Conclusion: We have begun to understand the HMV patterns that guide gene expression in both tissue/cell-type specific and ubiquitous manner.
\end{abstract}

\section{Background}

The development of a human body from a single fertilized egg is a spatially and temporally regulated complex process. The genes that are responsible for general cellular function are expressed in all cell-types and tissues. However, in many tissue/cell-types, specialized functions require or exclude the expression of certain genes. The mechanism of this tissue/cell-type specific regulation (TCSR) is rather intriguing. It is worth noting that such diverse expression patterns are achieved through one genome shared largely by all cells. Gene transcription is regulated in multiple layers, e.g. transcription factor binding through DNA nucleotide features, DNA methylations, and chromatin modifications. TCSR may involve

\footnotetext{
* Correspondence: michael.zhang@utdallas.edu

Department of Molecular Cell Biology, Center for Systems Biology, University of Texas at Dallas, 800 W Campbell Road, Richardson, TX 75080, USA
}

combinations of these regulations in all layers (for review [1-3]).

Thanks to next generation sequencing technology, our understanding of human TCSR has accelerated in recent years. At the base layer of DNA features, the association between DNA regulatory elements, such as TATA box and $\mathrm{CpG}$ islands in the promoter regions, and tissuespecific regulation has been investigated experimentally [1] and computationally [4]; Tissue-specific regulatory transcription factor binding sites in the promoter regions have been well studied in muscle [5] and liver [6], and binding sites were also detected in multiple tissues using generic transcription factor binding site prediction tools [7-9]. Cell-type specific enhancers have been experimentally explored in several cell types as well [10]. High-throughput Cap Analysis of Gene Expression (CAGE) data showed that alternative transcription start sites (TSS) exist in the mammalian

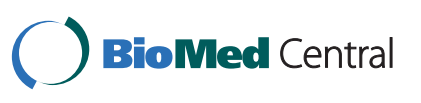


genome with more prevalence than previously thought [11], and, moreover, distributions of TSS have also been associated with TCSR [12]. Recently, genome-wide mapping of Histone Modifications and Variants (HMVs) in CD4+ T cells $[13,14]$, as well as other cell types [15], opened up an opportunity to model gene expression levels from the perspective of post-translational modification of histones [16]. For example, Pekowska et al. clustered genes by their H3K4me2 profile at the promoter regions in $\mathrm{CD} 4+\mathrm{T}$ cells. They found that a cluster was enriched in CD4+ T cell specific genes [17]. However, a comprehensive picture on how posttranslational modifications of histones contribute to TCSR is still not clear.

Therefore, in this work, we addressed three major questions 1) which HMVs carry sufficient information to allow TCSR target gene prediction, 2) whether TCSR is the same as gene expression activity regulation, and 3) whether the predictive relationship between HMV and TCSR target genes is universal for entire Pol II transcriptome. To properly address these questions, we developed a quantitative model to link the HMVs and TCSR target genes using CoreBoost, and applied it to recently published genome-wide mapped HMVs in CD4+ T cells $[13,14]$. CoreBoost is a previously developed boosting classifier $[18,19]$ that can select informative features from an ensemble of weak classifiers. We first show that HMV profiles in both proximal promoters and gene bodies are predictive for CD4+ $\mathrm{T}$ cell specificity. The most predictive HMV types have been identified for CpG- and nonCpG-related genes in promoters and gene bodies. The evidences have shown that the underlying enhancers and intragenic alternative promoters marked by the HMV patterns were associated with tissue/cell-type specific gene expression. Second, we demonstrated that TCSR is different from the regulation of gene expression activity. Finally, the model, which was trained on HMV data of protein-coding genes in CD4+ T cells, successfully predicted muscle cell specific genes and CD4+ $\mathrm{T}$ cell specific microRNA genes.

\section{Results and Discussion}

\section{Definition of $\mathrm{CD} 4+\mathrm{T}$ cell specific regulated genes}

We chose CD4+ T cells as the model, taking advantage of the widespread availability of genome-wide HMV data for this cell type $[13,14]$. CD4 $+\mathrm{T}$ cell specific expressed genes (denoted as CD4SE) and housekeeping genes (denoted as HK) were collected as positive and negative datasets. We identified CD4SE genes according to their expression profiles among human tissues and other information. Altogether, 454 and 630 genes were collected in CD4SE, and HK sets, respectively (see Methods and Materials).
Genes in the CD4SE set were not expressed in most tissue/cell-types other than blood cell types. We plotted the expression distribution of genes in CD4SE, HK and randomly selected genes among all tissues in the GNF symAtlas dataset [20] as shown in Additional file 1. CD4SE genes were only expressed in a small number of blood cell types (CD14, CD19, CD33, CD4, CD56, CD8, X721 B/T cells, and whole blood), as expected, since this result agrees with the high expression correlation between blood cells $[15,16]$. On the other hand, the HK genes and randomly selected genes were expressed in various tissue/cell-types studied. Quantitatively, both the overall entropy and categorical entropy in CD4+ T cells are significantly smaller in CD4SE genes than in HK genes [4] (the average overall entropies for CD4SE and HK genes are 4.8 and 6.26 as in the GNF symAtlas dataset $[4,20]$, respectively, $\mathrm{P}<2.2 \mathrm{e}-16$; the average categorical entropies for CD4SE and HK genes are 8.95 and 12.35 as in the GNF symAtlas dataset respectively, P < 2.2e-16).

\section{The predictive HMVs for CD4+ T cell specific regulation}

Previous studies suggested that CpG-and nonCpGrelated promoters have different regulatory characteristics [21-26], and have a contrasting distribution of HMVs [19]. Following the same strategy used previously for CoreBoost [18], CoreBoost_HM [19], and a third work [16], we analyzed CpG- and nonCpG-related genes separately. There were $40 \mathrm{HMV}$ types in the CD4+ T cell dataset $[13,14]$, many of which were correlated with each other [13]. We first performed a principal component analysis (PCA) and grouped the HMVs into two sets. For convenience, we refer to them as Set I and Set II. Set I contained the HMVs that have the highest contributions in the first 4 principal components (which captured $90 \%$ of variance, see Additional file 2). Set II contained the remaining HMVs. There were 25 and 15 HMVs in Set I and Set II, respectively. We trained CoreBoost to distinguish between CD4SE genes and HK genes. Because there were more genes in the HK set (630) than in the CD4SE set (454), we randomly sampled about 454 HK genes and combined them with CD4SE to form a total set. The performance of the CoreBoosts was evaluated based on sensitivity, positive predictive value (PPV) [27] and F-score [28]. Five-fold cross-validation was performed to limit over-fitting. To further eliminate any potential bias introduced by sampling fluctuation, we repeated the whole process 100 times.

We focused first on the features in proximal promoters. The classifiers trained with HMV in the proximal promoters significantly differentiate CD4SE genes from HK genes (Table 1). In both Set I and Set II, CoreBoost performed much better in nonCpG-related genes than 
Table 1 The performance of CoreBoost based on features in CpG- or nonCpG-related proximal promoter and gene body region

\begin{tabular}{|c|c|c|c|c|c|}
\hline HMV groups & & & Sensitivity & PPV & F-Score \\
\hline & Promoter & $\mathrm{CpG}$ & $0.579 \pm 0.016$ & $0.764 \pm 0.028$ & $0.658 \pm 0.006$ \\
\hline \multirow[t]{4}{*}{ Set I } & & non-CpG & $0.889 \pm 0.032$ & $0.771 \pm 0.021$ & $0.825 \pm 0.007$ \\
\hline & Body & $\mathrm{CpG}$ & $0.594 \pm 0.013$ & $0.789 \pm 0.021$ & $0.678 \pm 0.007$ \\
\hline & & non-CpG & $0.876 \pm 0.015$ & $0.811 \pm 0.013$ & $0.842 \pm 0.006$ \\
\hline & Promoter & CpG & $0.523 \pm 0.016$ & $0.717 \pm 0.021$ & $0.605 \pm 0.010$ \\
\hline \multirow[t]{3}{*}{ Set II } & & non-CpG & $0.908 \pm 0.033$ & $0.790 \pm 0.021$ & $0.844 \pm 0.007$ \\
\hline & Body & CpG & $0.588 \pm 0.015$ & $0.736 \pm 0.020$ & $0.653 \pm 0.008$ \\
\hline & & non-CpG & $0.892 \pm 0.014$ & $0.821 \pm 0.012$ & $0.854 \pm 0.006$ \\
\hline
\end{tabular}

Averages and errors are given as the mean and standard deviation from 100 replicates. All of the CoreBoost performance statistics have a p-value $<1$ - 5 .

in CpG-related genes. This is because CpG-related genes are more likely to be associated with housekeeping genes [4]. A few HMV types have been highlighted as the most predictive features in our 100 replicates (Figure 1 and Additional file 3 and 4), and we noticed that many of those selected features are located downstream of the TSS in both Set I and Set II (Additional file 5). We then plotted the distribution of the selected HMVs among promoter regions (Figure 2). We see that the major difference of the HMV levels between the CD4SE and HK promoters is found downstream of the annotated TSS, indicating that HMV patterns in gene bodies may also be predictive of TCSR targets.

To investigate this possibility, we designed a new HMV feature table containing the following information: the average and sum of each HMV level for the first exon and the first intron; the average and sum of each HMV level for the whole gene body; and the sum of each HMV level in the first twenty nucleosomes positioned after the first exon. The first exon and the first intron were chosen because previous studies had shown the first exon and/or intron can play important roles in

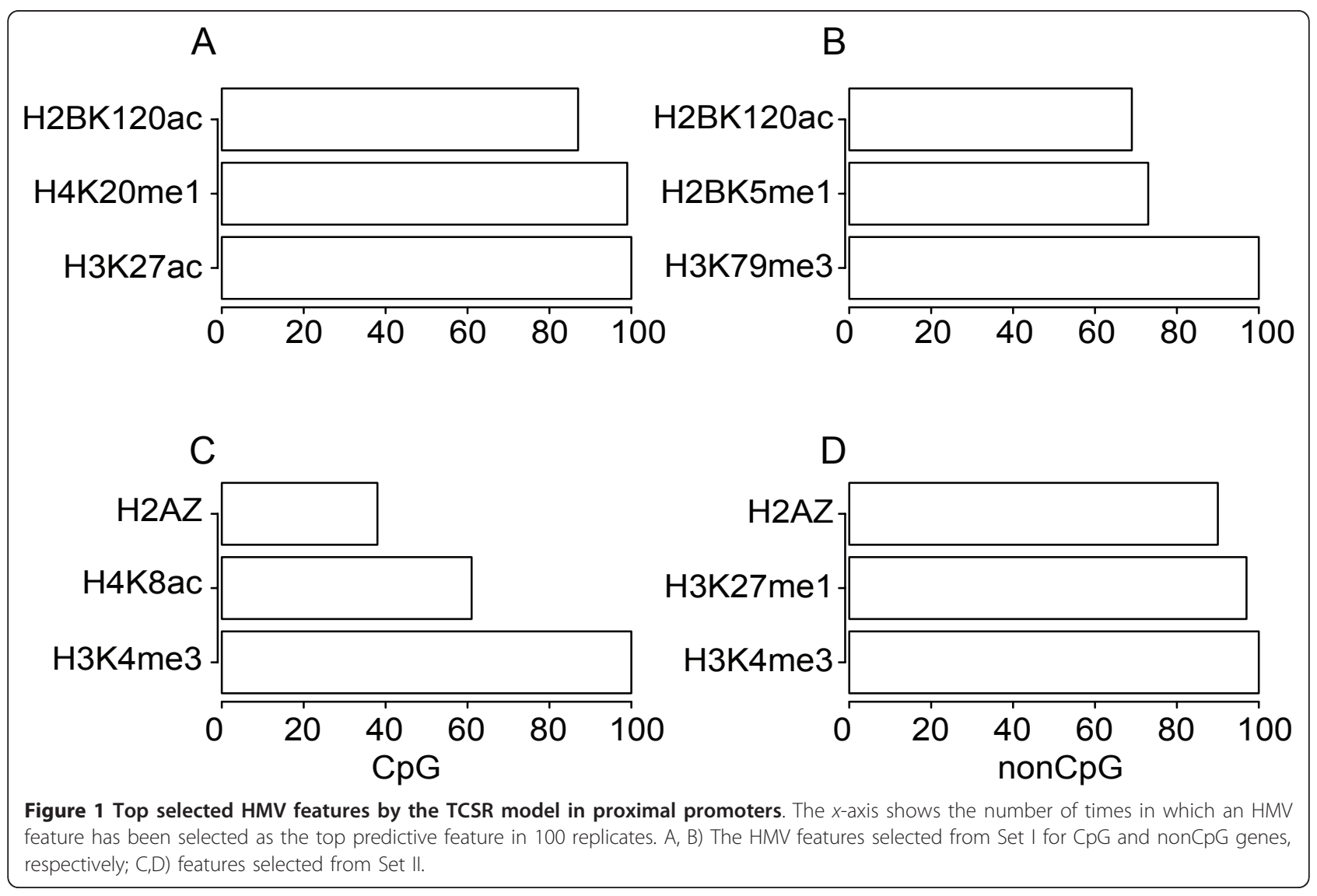




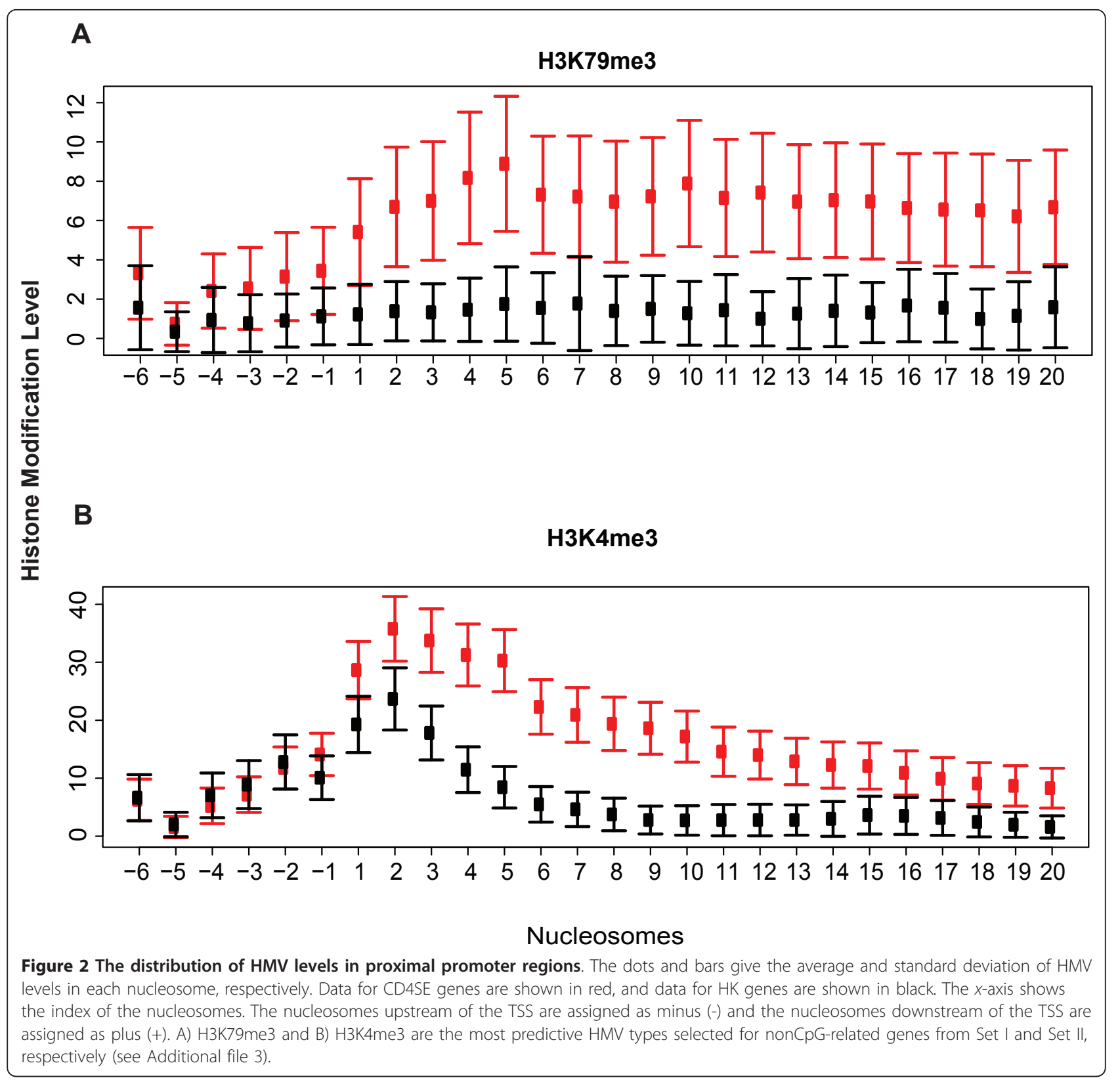

gene regulation [29], especially in tissue-specific regulation $[30,31]$. Using this newly designed feature table, we repeated the CoreBoost training and analysis. The "body" entries in Table 1 summarize the performance of the new CoreBoost classifier for 100 replicates. We found that the classifiers have similar performance, irrespective of whether the HMV features in promoter or in gene bodies were used for training, and both performed significantly better than classifiers trained by control regions (Table 1). For CpG-related genes, the features sums of H3K27ac, sums of H3K79me3 and sums of H3K4me3 levels in the entire gene bodies contributed most to the prediction of CD4+ T cell specificity (see
Additional file 5). For nonCpG-related gene, the features sums of H4K20me3 and sums of H3K14ac levels in the entire gene bodies contribute most to the prediction (see Additional file 5). Based on this line of evidence, we conclude that HMV profiles in gene bodies encode information about TCSR, much like those in promoters.

TCSR is different from gene expression activity regulation at the HMV level

We have shown above that TCSR target genes can be predicted by HMV profiles in both promoters and gene body regions. The immediate question that follows is how much gene expression level per se may determine 
TCSR. It might be argued our TCSR model achieves high predictive power is because CD4SE genes are highly expressed in CD4+ T cell and therefore could be easily predicted by any gene expression level prediction model. We now argue that this is not the case.

The predictive power of our TCSR model does not stem from the high expression level of CD4SE genes. First, if we define highly expressed genes as those genes whose expression levels are at least one standard deviation higher than average levels in a given cell type, then CD4SE genes are by no means highly expressed genes, even though they are higher than expression levels of HK genes (rank sum test $P=0.01$ ). Second, our model does not simply predict highly expressed genes as CD4+ $\mathrm{T}$ cell specific. For example, of the $159 \mathrm{CpG}$-related genes that were predicted as $\mathrm{CD} 4+\mathrm{T}$ cell specific in at least half of 100 replicates, only 26 genes were actually highly expressed in CD4+ $\mathrm{T}$ cell $(\mathrm{P}=0.005)$. Moreover, the predicted highly expressed genes by the model proposed by Karlic et al. are expressed in broad tissues, but our model predicted CD4+ $\mathrm{T}$ cell specific genes expressed only in limited blood cell types akin to CD4SE genes (Additional file 6). Therefore, it is not surprising that our predictions, in comparison, have significantly smaller overall entropies (average overall entropies are 5.7 and 4.3, respectively, rank sum test $\mathrm{P}<$ $2.2 \mathrm{e}-16)$ and categorical entropies (5.8 and 4.5, respectively, rank sum test $\mathrm{P}<2.2 \mathrm{e}-16$ ). The same observation can be made even if one removes the intersection of the two predictions (overall entropies are 5.7 and 4.3 respectively, $\mathrm{P}<2.2 \mathrm{e}-16$; and categorical entropies are 5.9 and 4.6 respectively, $\mathrm{P}<2.2 \mathrm{e}-16$ ).

The difference was also indicated by the distinct HMV types selected as predictive features between our TCSR model and the gene expression activity model proposed by Karlic et al. That is, while Karlic et al. identified HMV type pairs H4K20me1/H3K27ac and H3K4me3/ $\mathrm{H} 3 \mathrm{~K} 79 \mathrm{mel}$ as the most powerful predictive features for
CpG and nonCpG-related promoters respectively [16], our model identified different HMV type pairs for TCSR (Figure 1, Additional file 3 and 4). To explain, first, we noticed that the second most predictive features selected by CoreBoost for CpG promoters in Set I was H4K20me1 (Figure 1A), which was also one of the two most predictive features selected by Karlic et al for gene expression activity prediction. To ensure that the reason why we did not choose H4K20me1 as the most predictive feature was not because of the separation of features into two initial input sets, we retrained our model with the initial input features including all HMVs in the final selection of either models (H4K20me1, H3K27ac, H3K4me3, and H4K8ac for CpG-related promoters, and H3K79me3, H3K79me1, K3K4me3, H2BK5me1, and H3K27me1 for nonCpG-related promoters, see Table 2). Indeed we still found that $\mathrm{H} 3 \mathrm{~K} 4 \mathrm{me} 3 / \mathrm{H} 3 \mathrm{~K} 27 \mathrm{ac}$ and H3K4me3/H3K79me3 remain to be selected by our CoreBoost model as the best predictors of TCSR for CpGand nonCpG-related promoters, respectively. Second, it is known that some HMVs are highly correlated, for example H3K27ac and H2BK5ac are highly correlated in both CD4SE and highly expressed genes (median values of $r$ are 0.91 and 0.78 , respectively), but H3K23ac and H4K20me1 are not highly correlated (median values of $r$ are 0.1 and 0.09 , respectively). Therefore, if the HMV types selected by the two models are truly different, we should expect at least one pairing of HMV types from either model to be poorly correlated in their promoter regions. We compared the Pearson correlation coefficient of the HMV profiles in each case for both CD4SE and highly expressed gene promoters (Table 2). For the HMV type pairs selected by our TCSR model, there is always an HMV type that has low correlation with both of the HMVs selected by the model of Karlic et al. This is more so in nonCpG-related promoters than CpG promoters. As we discussed above this is probably related to the fact that CpG-related genes are, in general, largely

Table 2 The correlations between predictive HMV profiles in nonCpG-related and CpG-related promoters

\begin{tabular}{|c|c|c|c|c|c|c|}
\hline & & & \multicolumn{2}{|c|}{ CD4SE } & \multicolumn{2}{|c|}{ High } \\
\hline & & & H3K4me3 & H3K79me1 & H3K4me3 & H3K79me1 \\
\hline \multirow[t]{4}{*}{ non-CpG } & Set I & H3K79me3 & 0.346 & 0.344 & 0.190 & 0.420 \\
\hline & & $\mathrm{H} 2 \mathrm{BK} 5 \mathrm{me} 1$ & 0.021 & 0.295 & -0.072 & 0.378 \\
\hline & Set II & H3K4me3 & 1.000 & 0.161 & 1.000 & 0.127 \\
\hline & & H3K27me1 & 0.006 & 0.231 & 0.014 & 0.355 \\
\hline \multirow[t]{5}{*}{ CpG } & & & H4K20me1 & H3K27ac & H4K20me1 & H3K27ac \\
\hline & Set I & H4K20me1 & 1.000 & 0.032 & 1.000 & -0.009 \\
\hline & & H3K27ac & 0.032 & 1.000 & -0.009 & 1.000 \\
\hline & Set II & H3K4me3 & 0.121 & 0.688 & -0.013 & 0.799 \\
\hline & & H4K8ac & -0.031 & 0.512 & -0.121 & 0.748 \\
\hline
\end{tabular}

Two of the most frequently selected HMV types by our CoreBoost model in Set I and Set II are represented in rows for each subset. The two HMV types selected by the model of Karlic et al are represented in columns. The numbers are the median values of Pearson's correlation coefficient of HMV profiles in the promoter of CD4SE genes or promoter of highly expressed genes. 
housekeeping genes. Therefore, the most predictive HMV types which were chosen for TCSR prediction by CoreBoost and the HMV types which have been chosen for gene expression activities by the model of Karlic et al were true dissimilar.

\section{What makes HMV predictive for tissue/cell-types specific regulation?}

We noticed that H3K4me3 had been chosen as the most predictive HMV marks in both the promoter and gene body region for both CpG- and nonCpG-related genes by our TCSR model. H3K4me3 is a well-documented HMV signal that marks the promoter [13,14,25,32-34]. This fact let us to investigate the roles of intragenic alternative promoters for TCSR. To infer the potential promoter activities in gene bodies, we further looked at the capped analysis of gene expression (CAGE) experimental data and DNA methylation data in the gene bodies. Because it is well known that 1) CAGE data directly indicates the transcription initiation site [11], and 2) DNA methylation in promoters suppresses gene expression [35], we used the two datasets as positive and negative controls, respectively. If the alternative promoters in the gene body contribute to TCSR, we should expect to observe relatively higher H3K4me3 levels and CAGE tags in the gene body of tissue/cell specific genes than those of housekeeping genes. On the other hand, a relatively higher DNA methylation level should be observed in gene bodies of housekeeping genes. In the ENCODE project [36], the Broad Institute mapped HMVs for the K562 cell line [24], the RIKEN Institute did CAGE experiments on the same cell line [37], and the DNA methylation level has also been measured in the cell line via Methyl-seq technology by Brunner and colleagues [38]. We identified the K562 specific expressed genes in a manner similar to that used for CD4+ T cells, and all three datasets were compared between $\mathrm{K} 562$ specific genes and HK genes in the gene body (Figure 3A). As expected, intragenic H3K4me3 levels are significantly higher in K562 specific genes than in HK genes $(\mathrm{P}<0.05)$. Furthermore, intronic CAGE levels are higher in K562 specific genes than in HK genes $(\mathrm{P}<0.001)$, while exonic DNA methylation levels are generally lower in K562 specific genes than in HK genes $(\mathrm{P}<0.05)$. The association between DNA methylation and promoter is weaker and has longer physical distance in DNA sequences than is CAGE, because the mechanisms by which DNA methylation to suppresses gene expression are circuitous in nature, e.g., by recruitment of methyl-CpG-binding domain proteins (MBD), which, in turn, recruit histone modifying and chromatin-remodeling complexes to the site to change the histone status (for review see [39]). Nevertheless, the data we showed here indicated that the alternative promoters in gene body could associate with TCSR. For example, troponin I type 3 (cardiac), TNNI3, is a gene specifically expressed in heart (z-score of categorical entropy $>13$ ) and Leukemia cell lines (the $\mathrm{z}$-score $>2.8$ for K562). We saw strong CAGE peaks in the intronic regions. There are CAGE tag peaks found in the third and the fourth introns which have been further marked by high H3K4me3 levels. An unmethylated CpG island covered the region from the third exon to the fifth exon. This corroborating data strongly suggests that specific expression of TNNI3 in K562 could be regulated via alternative promoters located in the third and the fourth introns (Figure 3B). Interestingly, Alika and colleagues recently reported that alternative promoters in the gene body of SHANK3 regulate human brain specific expression of the gene [40]. The alternative promoters in SHANK3's gene body were marked by high level of H3K4me3, as well as CAGE tags and unmethylated CpG islands.

There are several other possible associations between TCSR and HMV patterns. The HMV patterns could be markers in the nucleosomes indicating enhancers in the nearby DNA sequence. The binding of a transcription regulatory factor at an enhancer has long been suggested as one of the most important mechanisms of tissue/cell-type regulation [10,15,34]. H3K4me1 is most frequently associated with enhancers $[10,13]$. We compared H3K4me1 profiles in the gene body with the profile of other HMV types (see Additional file 7). For the 15 HMVs which most correlated with H3K4me1, 13 of them (87\%, hypergeometric test $\mathrm{P}=9.3 \mathrm{E}-9)$ were selected as the top predictive features by resampling at least once in the 100 replicates. In addition, there are other HMVs types associated with the enhancers. For example, H2A.Z, H3K27ac, monomethylated H3K4, $\mathrm{H} 3 \mathrm{~K} 9$, and H3K27 were all found to be strongly associated with enhancers [13-15,32,41,42]. Also, six HMVs (H3K4me1, H3K4me2, H3K4me3, H3K9me1, H3K18ac, and H2A.Z) were detected at more than a fifth of potential enhancers [13]. All of these HMVs were selected as predictive HMVs at least once by resampling (see Additional file 5), indicating the possibility of the underlying enhancer activity in the regions.

Another possibility is that tissue/cell-type specific expression could be regulated after transcription initiation and/or in the pause and elongation stages. Recent studies implied that the majority of genes are transcriptionally initiated and paused [43-45]. H3K79me2, a characteristic marker of RNAPII elongation, is only found downstream of TSS in the human genome [46]. In our data, H3K79me2 is a most frequently selected predictive HMV among the 100 replicates from Set I (see Additional file 4). In nearly all the cases (except for the Set I HMVs in the nonCpG related promoters), as shown in 


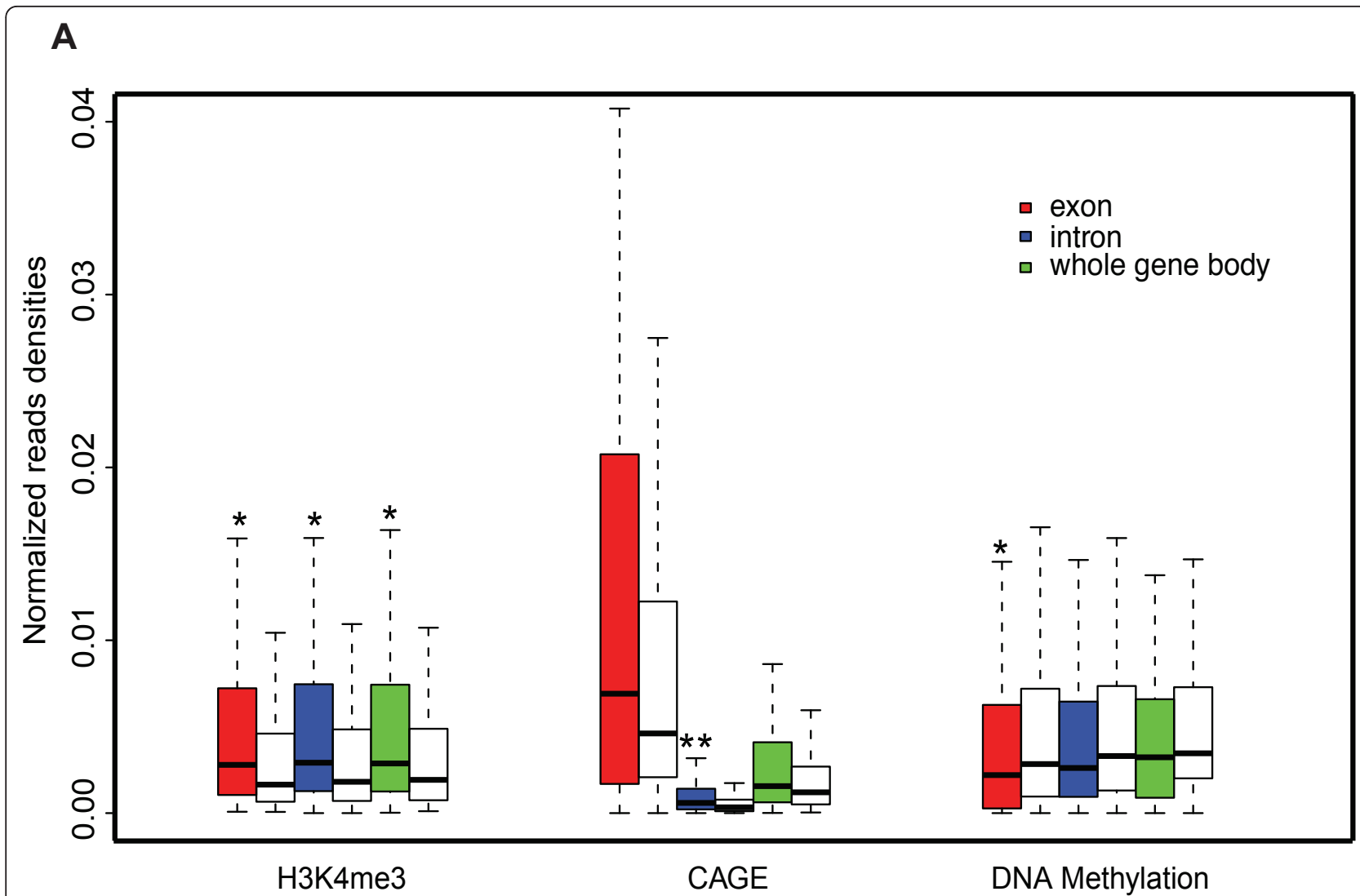

B

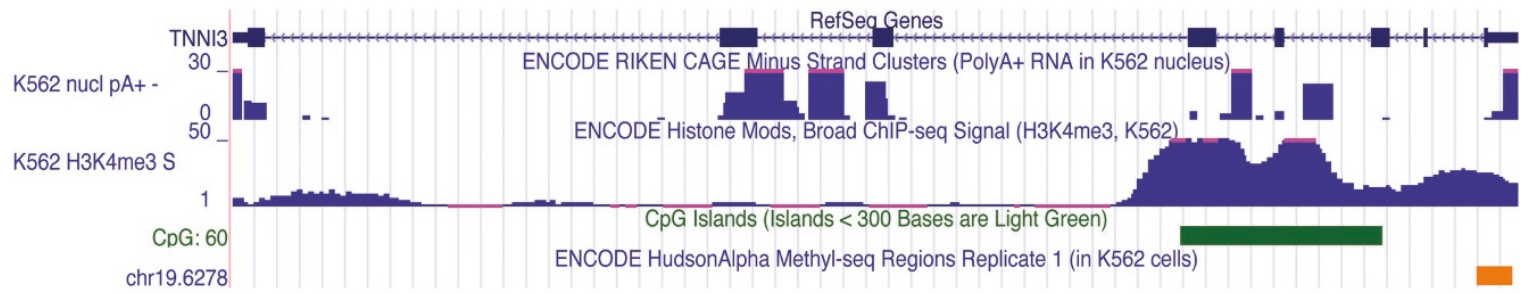

Figure 3 Alternative promoter activities in the gene body. A) H3K4me3, CAGE tag and DNA Methylation levels were compared between K562 specific genes and housekeeping genes in the K562 cell line. All data were controlled by region lengths. H3K4me3 and CAGE tag levels have been further controlled by gene expression levels. DNA methylation levels have been further controlled by CpG island lengths. Red, blue, and green boxes represent data in exonic, intronic, and whole gene body regions for K562 specific genes respectively. Blank boxes represent data in housekeeping genes for the same type of regions as the box next to its left. The Wilcoxon rank sum test P-values between the K562 specific genes and housekeeping genes are shown by the ${ }^{\prime * \prime}(P<0.05)$ and ${ }^{\prime * * \prime}(P<0.0001)$; B) Detailed data of H3K4me3, CAGE, and DNA methylation density for K562 specific gene TNN/3.

Table 2, we noticed an HMV highly correlated with $\mathrm{H} 3 \mathrm{~K} 4 \mathrm{me} 3$ (in nonCpG related genes) and H3K27ac (in CpG-related genes), respectively. H3K4me3 and H3K27ac are well-known gene activity markers [13,14]. The other HMVs are much less correlated with either of the HMVs selected by the gene activity model Karlic et al (except for the Set II HMVs in the CpG related promoters). Given this observation, we propose that the
HMV profile of H3K27ac and H3K4me3, together with other correlated HMV types, may provide a basal layer of information for gene transcriptional regulation in CpG- and nonCpG-related genes, respectively. And as additional signals, the remaining HMV marks may be "modulated" on top of the basal signals so that the tissue/cell-type specificities of gene expression can be achieved. This modulation process could be manifested 
either by guiding the binding of transcription factors at enhancer regions or by directing the pause or elongation of transcription, as discussed above.

\section{The HMV profile marks skeletal muscle myoblasts specific genes}

We next asked whether the predictive HMV model trained by the CD4+ T cell data could also be used to predict in other cell types. We collected HMV data for normal human skeletal muscle myoblasts (HSMM) from the ENCODE project [36], in the Broad Institute Chipseq dataset [24]. A total of 416 HSMM specific expressed genes were identified by the same method as used for CD4+ $\mathrm{T}$ cells. There were eight HMV types (H3K4me1, H3K4me2, H3K4me3, H3K9ac, H3K27ac, H3K27me3, H3K36me3, and H4K20me1) available in the ENCODE dataset. CoreBoost classifiers were retrained based on these eight HMV types in the CD4+ $\mathrm{T}$ cell data. By applying these newly trained classifiers to the HSMM input data, the classifiers successfully predicted HSMM specific genes with similar sensitivities as before with the CD4+ T cell input data (Table 3). However, the specificities (PPV) of the new classifiers were lower than before for $\mathrm{CpG}$ related genes (Table 1), possibly because CpG related genes are more likely to be housekeeping genes. Nevertheless, the performance of these newly trained classifiers are significant better than the controls, which were also retrained based on the eight HMV types in the CD4+ T cell data in the control regions and applied to HSMM dataset (Table 3). Therefore, the model we trained by the CD4 $\mathrm{T}$ cell data was not specific to the CD4 $\mathrm{T}$ cells, and it can be applied to other cell types as well.

\section{Prediction of CD4+ T cell specific regulation of microRNA genes}

MicroRNAs (miRNA) are a class of short RNA molecules which are generated from intergenic or intronic transcripts called pri-miRNAs (for review see $[47,48]$ ).
Similar to mRNA, pri-miRNAs also have a 5' cap structure and a 3' ployA tail [49]. The majority of pri-miRNAs are believed to be transcribed by Pol II [50], with a few exceptions [51]. Nevertheless, most pri-miRNAs share a transcription mechanism similar to protein-coding genes.

To test whether the association between TCSR and HMV patterns we found in protein-coding gene is similar for miRNAs genes, we trained our CoreBoost classifiers using the HMV profiles of protein-coding genes and applied them to miRNA genes. We evaluated our prediction with a recently published miRNA expression atlas [52] in which 13 and 50 CpG-related miRNAs clusters were identified as $\mathrm{CD} 4+\mathrm{T}$ cell specific and housekeeping, respectively. The performance of the classifiers trained in promoter and gene body was significantly better than the performance of classifiers trained in control regions (Table 3), although they were not as good as the performance for predicting protein-coding genes (Table 1). The relatively lower performance of the classifiers on miRNA most likely results from the fact that we do not have sufficient knowledge about the miRNA gene structures, e.g., the TSS, the full length of pre-miRNA transcript, or the existences or the lengths of first exon/introns. The promoter regions of miRNA genes used for this prediction were obtained by recent computational predictions [19]. However, because of the shortage of high-quality training data, miRNA promoter prediction is a difficult problem, and the resolution and the accuracy of the predictions are relatively lower $[19,23]$. On the other hand, our classifiers were trained on the HMV profiles in individual hypothetical nucleosomes related to a well-defined TSS. Thus, the low resolution of promoter prediction has a significant effect on the nucleosomes assignment (as 500-bp resolution could end up with a difference of about 3 nucleosomes). This effect lowers the expectation of the predictive power of our HMV promoter trained classifiers. Nevertheless, even without full knowledge, our model was still be able

Table 3 The performance of CoreBoost classifiers

\begin{tabular}{|c|c|c|c|c|c|}
\hline & & & Sensitivity & PPV & F-Score \\
\hline \multirow[t]{4}{*}{ HSMM } & Promoter & CpG & $0.929 \pm 0.098$ & $0.467 \pm 0.012$ & $0.619 \pm 0.033$ \\
\hline & & non-CpG & $0.764 \pm 0.214$ & $0.617 \pm 0.043$ & $0.662 \pm 0.123$ \\
\hline & Body & $\mathrm{CpG}$ & $0.919 \pm 0.135$ & $0.473 \pm 0.022$ & $0.618 \pm 0.043$ \\
\hline & & non-CpG & $0.860 \pm 0.183$ & $0.608 \pm 0.025$ & $0.700 \pm 0.093$ \\
\hline \multirow[t]{4}{*}{ miRNA } & Promoter & Set 1 & $0.417 \pm 0.037$ & $0.441 \pm 0.036$ & $0.426 \pm 0.011$ \\
\hline & & Set II & $0.365 \pm 0.073 \dagger$ & $0.521 \pm 0.085$ & $0.422 \pm 0.079+$ \\
\hline & Body & Set 1 & $0.255 \pm 0.080$ & $0.220 \pm 0.068 \dagger$ & $0.234 \pm 0.068+$ \\
\hline & & Set $\|$ & $0.125 \pm 0.057^{*}$ & $0.099 \pm 0.069^{*}$ & $0.102 \pm 0.056^{*}$ \\
\hline
\end{tabular}

In the "HSMM" rows, the classifiers were trained based on eight HMV features in CD4+ T cells, in the proximal promoter and gene body region, respectively. In the "miRNA" rows, the classifiers were trained on full Set I or Set II HMV features. Averages and errors are given as the mean and standard deviation from 100 replicates. The significances of comparison between the performance of CoreBoost trained on features in those regions and control regions are indicated by symbols next to each number. No symbol indicates $p$-value $<1 e-5$; ${ }^{*}$ indicates $p$-value $<1 e-2$ and $>=1 e-5$; indicates $p$-value $>1 e-2$. 
to correctly predict about $40 \%$ of CD4+ T cell specific miRNAs, and this prediction was significantly better than the control. This result suggested that miRNA genes may share a similar association between HMV patterns and TCSR with protein-coding genes.

\section{Predictive information is redundantly distributed among HMVs}

In this work, we identified H3K4me3, H3K79me3, and $\mathrm{H} 3 \mathrm{~K} 27 \mathrm{ac}$ as the most predictive marks in the promoter regions (Figure 1). However, these three HMV marks are by no means the only predictive ones. For example, H3K79me2 has also been selected as the most predictive HMV marks in nonCpG-related gene bodies (see Additional file 4). Therefore, we can reasonably argue that the predictive power for detecting TCSR targeted gene is redundantly encoded among HMVs. One clue indicating the existence of such redundancy was the success of applying our model to HSMM cell input data. Instead of using the full model, we trained our CoreBoost classifiers with the eight HMV types which were available in the ENCODE dataset. Although neither H3K79me2 nor H3K79me3 were available in the ENCODE dataset, the classifiers still managed to make significant predictions with similar performance as those trained with the full HMV set (Table 3).

To further exclude the possibility that this high performance could not be attributed to the existence of one or several dominating HMV marks, we performed the training and testing once more with a subset of HMV type set, in which all three of the most predictive HMV types H3K4me3, H3K79me3, and H3K27ac were removed. We also excluded H3K4me2 from the training data because this HMV type has recently been suggested as a unique mark for $\mathrm{CD} 4+\mathrm{T}$ cell specificity [17]. Interestingly, the classifiers also achieved similar significant predictive power as the classifiers trained by the full HMV profile (Table 3 and Additional file 8). With the possible exception of H3K9me1, Pekowska et al. did not find any other HMV marks than H3K4me2 that could make the same enrichment of CD4+ T cell specific genes [17]. This is probably because clustering did not fully reveal the profound relationship between HMV profile and TCSR. To explore this possibility, we revisited the cluster (cluster 1 ) in which they observed enrichment of CD4+ T cell specific genes. By comparing the entropies between cluster 1 and CD4SE by using the GNF symAtlas dataset, we found that the overall entropy of cluster 1 was larger than CD4SE (5.5 and 4.8 respectively, $\mathrm{p}<2.2 \mathrm{e}-16)$, and that categorical entropy was also larger than CD4SE (10.8, and 8.95 respectively, $\mathrm{p}<2.2 \mathrm{e}-16)$, implying that the genes in the cluster 1 are significantly less specific to CD4+ T cells than the genes in CD4SE. Only 66 out of 392 genes in the cluster 1 were actually CD4+ T cell specific expressions according to our definition of TSCR by gene express entropy (sensitivity $=0.14, \mathrm{PPV}=0.16$ and F-score $=0.15$ ).

\section{Conclusions}

We have utilized CoreBoost to connect the HMV and TCSR patterns in CD4+ T cells. From this data we draw the following conclusions. First, we found that patterns of HMV contain sufficient information to predict TCSR target genes. The classifier we trained on HMV data successfully distinguished CD4+ T cell specific genes from housekeeping genes. Predictive HMV information was not only found in promoter regions, but also in the gene body. This finding is important because it implies the existence of multiple regulatory elements which could be marked by HMVs for TCSR. Second, we identified predictive HMV marks for $\mathrm{CpG}$ - and nonCpGrelated genes. In promoters, $\mathrm{H} 3 \mathrm{~K} 4 \mathrm{me} 3$ and $\mathrm{H} 3 \mathrm{~K} 27 \mathrm{ac}$ were the most predictive HMV marks for CpG-related genes, whereas for nonCpG-related genes H3K4me3 and H3K79me3 were the most predictive. However, even if we excluded data from the most predictive HMV marks, we found that the remaining data still have sufficient predictive ability to make significant predictions for TCSR target genes. This information redundancy again points to the existence of multiple regulatory elements which could be marked by HMVs for TCSR. By carefully surveying patterns of $\mathrm{HMV}$, we further propose that marking the underlying enhancers and marking intragenic alternative promoters are two potential mechanisms that could guide TCSR. Finally, we provide evidence showing that TCSR in other tissue/cell-types, as well as TCSR for non-protein coding Pol II transcripts, such as microRNA, may share TCSR HMV patterns similar to the case of $\mathrm{CD} 4+\mathrm{T}$ cells. The associations between the HMV patterns and TCSR we found may be generic, as we successfully predicted genes with muscle cell specific expression, as well as microRNA genes with CD4+ T cell specific expression, by the same classifier which was trained on the HMV data of protein-coding genes in $\mathrm{CD} 4+\mathrm{T}$ cells.

\section{Methods}

\section{Data}

The RefSeq Gene annotation track for the human genome sequence (hg18) was downloaded from the University of California Santa Cruz Genome Browser (UCSC, http:// genome.ucsc.edu/). The exon information was downloaded from BioMart at Ensembl (http://www.ensembl. org/biomart/). Two gene expression data sets in human tissues were taken from the GNF symAtlas database [20]; and the GEO database (http://www.ncbi.nlm.nih.gov/geo/, GSE7307). We defined a promoter to be CpG-related if there was a CpG island located within its upstream $2 \mathrm{~kb}$ to 
downstream 500-bp region from the TSS [53]. The CpG island annotations were downloaded from the UCSC Genome Browser as well. HMV data for $\mathrm{CD} 4+\mathrm{T}$ cell were retrieved from genome-wide studies of the distribution of 19 lysine or arginine histone methylations and H2A.Z histone variant [14], and mapping of 19 histone acetylation [13]. HMV data for normal human skeletal muscle myoblasts (HSMM) and K562 cell lines were retrieved from the ENCODE project [36], specifically in the Broad Institute Chip-seq dataset [24]. In addition, as part of the ENCODE project, CAGE experimental data for the K562 cell line were retrieved from the RIKEN institute [37], and DNA methylation level in K562 were retrieved from the work of Brunner and colleagues [38]. The miRNA expression profiles were retrieved from a small RNA library based sequencing atlas [52]; we used the number of clones for each miRNA cluster to represent the expression of the pri-miRNA in each tissue. The promoter of a miRNA cluster was chosen as the closet promoter predicted for the members in the cluster [19].

\section{Identifying tissue-specific and housekeeping transcripts}

As a measurement of information content, Shannon entropy has been used for measuring the tissue-specificity of gene expression [4]. As the information content (tissue-specificity) of a distribution increases, its entropy decreases. Borrowing this concept, we measured the CD4 $+\mathrm{T}$ cell specific expressed gene set by the combination of the following two datasets: 1 ) genes that the overall gene expression entropy is smaller than 5.0 and categorical entropy less than 9 [4]; and 2) manually selected genes. From the literature, we manually selected 40 genes that play certain roles in CD4+ T cell development or maturation (see Additional file 9). The combination of the above two datasets contains 454 genes (see Additional file 9). The housekeeping genes were defined according to two criteria: 1) the overall gene expression entropy larger than 6.2 by GNF symAtlas dataset $[4,20]$; 2) the overall gene expression entropy larger than 8.9 by GSE7307 dataset. In total, there were 630 genes identified as housekeeping genes (see Additional file 9). The threshold for CD4+ T cell specificity was determined according to the bell shape distribution of categorical entropy ( $\mathrm{z}$ score $>2$ ). The threshold for housekeeping genes was determined as the one at the turning point of the overall entropy distribution curve, which has an exponential-like shape. We also tried several thresholds surrounding values, and we retrained our model accordingly, but no significantly different results were observed.

\section{Definition of feature tables for promoter and gene body regions}

The promoter region was defined as the region from the 6th nucleosome upstream of the transcription start site
(TSS) to the 20th nucleosome downstream of the TSS. We adopted the position definitions of -2 to +5 nucleosomes relative to the TSS used by Dustin et al. [2](-2[-370: -196], -1[-195: -46], +1 [-45:134], +2 [135:314], +3 $[315: 494],+4[495: 674]$ and +5 [675:859]). For the positions of other nucleosomes, we simply extended $150 \mathrm{bp}$ from its immediate neighbor nucleosomes. We tried another combinations of up- and downstream nucleosome numbers to define the promoter region (from the 6th nucleosome upstream of the TSS to the 9th downstream of the TSS), but it did not change the results. To construct the feature table for a gene, HMV levels were individually calculated for each HMV type. For any given HMV type, the sum of the HMV tag numbers in a nucleosome was assigned as the HMV level on that nucleosome. The HMV feature of a gene is therefore an array containing all HMV levels of each nucleosome within the proximal promoter. Taken together, there are $40 \mathrm{HMV}$ levels (including the bound level of the CCCTC-binding factor) on all 26 nucleosomes for each gene. The feature table for gene body regions is defined in the main text.

\section{PCA analysis was performed by using $R$}

The sum of tags in the $4 \mathrm{k}$ region around the TSS ([-2k, $+2 \mathrm{~K}]$ ) of all HMVs for all genes forms a matrix in which rows represent genes and columns represent HMV types. PCA analysis produced the linear combinations of columns. The first 4 principal components were chosen to form Set I and the remaining HMVs belonged to Set II.

\section{CoreBoost and performance evaluations}

CoreBoost is a boosting technique with stumps [18]. Boosting is a supervised machine learning algorithm which combines a group of weak classifiers to form a single strong classifier [54]. Stumps are single-split decision trees with only two terminal nodes [55]. The informative features are selected by CoreBoost to build the strong classifier (for more details, see Zhao et al. [18]). The performance of CoreBoost classifications was evaluated by sensitivity, positive predictive values (PPV, [27]), and F-score [28], which are defined as

$$
\begin{aligned}
& \text { Sensitivity }=\frac{T P}{T P+F N}, \\
& P P V=\frac{T P}{T P+F P}, \\
& \text { Fscore }=\frac{2 \times \text { Sensitivity } \times P P V}{\text { Sensitivity }+P P V},
\end{aligned}
$$

where TP denotes true positives, TN denotes true negatives, FP denotes false positives and FN denotes false negatives. 


\section{5 -fold cross validation}

The evaluations and the number of features for selection by CoreBoost were all obtained by 5 -fold cross validations. The procedure was as follows: Given a total dataset $D, 1) D$ was randomly partitioned into 5 subsets $D_{i}(i$ $=1,2, \ldots, 5) ; 2$ ) each $D_{i}$ was removed exactly once from $D$ and a CoreBoost classifier was trained on the remaining $80 \%$ and tested on the removed $D_{i}$; and 3) the final evaluations were the average of tests on 5 subsets. The final classifier was trained on total dataset $D$, which was used to predict the miRNAs expressed specifically in CD4+ T cells.

\section{The definition of control regions}

We chose a position that was $50 \mathrm{~kb}$ upstream of any given annotated TSS as its control site. When we tried same control with $500 \mathrm{~kb}$, we observed essentially the same results.

\section{Additional material}

Additional file 1: The distribution of gene expressions across tissues. The names of tissues are the same as those shown in the GNF symAtlas dataset. $A, B, C$ ) show the distributions for CD4SE, HK, and randomly chosen genes, respectively.

Additional file 2: The screeplot of the principal component analysis The screeplot of principal component analysis, where the $x$-axis gives the index of each principal components, and the $y$-axis gives the proportion of variance.

Additional file 3: Top selected HMV features by the TCSR model in gene bodies. Top selected HMV features by the TCSR model in gene bodies. The $x$-axis shows the number of times in which an HMV feature has been selected as the top predictive feature in 100 replicates. A, B) The HMV features selected from Set I for CpG and nonCpG genes, respectively; C, D) features selected from Set II.

Additional file 4: Top bi-combinations of selected HMVs features by the TCSR model. Top bi-combinations of selected HMVs features by the TCSR model. The $x$-axis shows the total number of times in which the two HMV types have been selected as the first and the second most predictive feature in 100 replicates, irrespective of nucleosomes index of the HMV features. The $y$-axis indicates the combinations of two HMV types in the "first_second" order. A, B) The combinations selected from Set I for CpG- and nonCpG-related promoters, respectively; C, D) combinations selected from Set II for CpG-and nonCpG-related promoters, respectively; $E, F)$ The combinations selected from Set I for CpG- and nonCpG-related gene bodies, respectively; G, H) combinations selected from Set II for CpG- and nonCpG-related gene bodies, respectively.

Additional file 5: Top selected HMV features by the TCSR model Top selected HMV features by the TCSR model. The $x$-axis shows the number of times in which a HMV feature has been selected as the top two predictive HMV features in 100 replicates. $p$ and $m$, stand for " + " and "-" strands, respectively, followed by an index of the nucleosome, either in downstream or upstream of TSS. "avg" means the average tag number of the HMV type; "body", "1stExon", and "1stintron" means that the calculation was performed in the entire gene body region, the first exon, and the first intron region, respectively. A, B) HMVs were selected from Set I for CpG- and nonCpG-related promoters, respectively; C, D) HMVs were selected from Set II for CpG- and nonCpG-related promoters, respectively; E, F) HMVs were selected from Set I for CpG- and nonCpG related gene bodies, respectively; C, D) HMVs were selected from Set II for CpG- and nonCpG-related gene bodies, respectively.
Additional file 6: The distribution of gene expression across tissues The distribution of gene expression across tissues. The name of tissues are the same as shown in the GNF symAtlas dataset. A) The TCSR model predicted CD4+ T cell specific genes. B) Predicted highly expressed genes in CD4+ $T$ cells based on the gene expression activity model of Karlic et al.

Additional file 7: The HMV types highly correlated with enhancer marker H3K4me1. All HMVs types with a Pearson's correlation coefficient compared with H3K4me1 higher than 0.2 in CD4SE genes are listed in here. The HMV names in bold font indicate they have been selected as a top predictive feature by CoreBoost at least once in 100 replicates.

Additional file 8: The performance of classifiers. The classifiers were trained by the HMV profile subset of CD4+ T cell, in which H3K4me3, H3K4me2, H3K79me3, and H3K27ac were not included. Averages and errors are given as the mean and standard deviation, respectively, from 100 replicates. The performances were measured by applying the classifiers on protein-coding genes in CD4+ T cells. The significances of comparison between the performance of CoreBoost trained on features in those regions and control regions are indicated by symbols next to each number No symbol indicates p-value < 1e-5; * indicates p-value < $1 \mathrm{e}-2$ and $>=1 \mathrm{e}-5$; indicates $\mathrm{p}$-value $>1 \mathrm{e}-2$.

Additional file 9: Gene name list. This file lists the CD4+ T cell specific and housekeeping protein-coding genes and miRNA genes.

\section{List of abbreviations}

HMV: histone modification/variation; TCSR: tissue/cell-type specific regulation; TSS: transcription start site; CAGE: Cap Analysis Gene Expression; PPV: positive prediction values; $\mathrm{H} 3 \mathrm{~K} 4 \mathrm{me} 3$ : histone $\mathrm{H3}$ trimethylated at lysine 4; H3K4me2: histone $\mathrm{H3}$ dimethylated at lysine 4; H3K4me1: histone $\mathrm{H} 3$ monomethylated at lysine 4; H3K27ac: histone $\mathrm{H3}$ acetylated at lysine 27; H3K79me2: histone H3 dimethylated at lysine 79; H3K79me3: histone H3 trimethylated at lysine 79; H3K9ac: histone $\mathrm{H3}$ acetylated at lysine 9; H3K27me3: histone $\mathrm{H3}$ trimethylated at lysine 27; H3K36me3: histone $\mathrm{H3}$ trimethylated at lysine 36; H4K20me1: histone $\mathrm{H} 4$ mono-methylated at lysine 20.

\section{Acknowledgements}

We thank Dr. Piero Carninci and Dr. Kawaji Hideya of RIKEN, Japan for providing early access to the Fantom data. We thank Dr. Monica Sleumer, Dr. Greg Vatcher, Dr. Jeff De Jong, Will Liao, Dr. Pradipta Ray, and David Martin for proofreading of the manuscript. We thank the two anonymous reviewers for their suggestions. This work was supported by a NIH R01 grant (HG001696) to M.Q.Z.

\section{Authors' contributions}

$\mathrm{ZZ}$ and $M Z$ designed the project. ZZ performed the experiment and data analysis. ZZ and $M Z$ wrote the paper; both authors read and approved the final manuscript.

Received: 9 December 2010 Accepted: 14 May 2011

Published: 14 May 2011

\section{References}

1. Maston GA, Evans SK, Green MR: Transcriptional regulatory elements in the human genome. Annu Rev Genomics Hum Genet 2006, 7:29-59.

2. Schones DE, Cui K, Cuddapah S, Roh TY, Barski A, Wang Z, Wei G, Zhao K: Dynamic regulation of nucleosome positioning in the human genome. Cell 2008, 132(5):887-898.

3. Allis CD, Jenuwein T, Reinberg D: Epigenetics. Cold Spring Harbor, New York: Cold Spring Harbor Laboratory Press; 2007.

4. Schug J, Schuller WP, Kappen C, Salbaum JM, Bucan M, Stoeckert CJ Jr: Promoter features related to tissue specificity as measured by Shannon entropy. Genome biol 2005, 6(4):R33.

5. Wasserman WW, Fickett JW: Identification of regulatory regions which confer muscle-specific gene expression. J Mol Biol 1998, 278(1):167-181. 
6. Krivan W, Wasserman WW: A predictive model for regulatory sequences directing liver-specific transcription. Genome Res 2001, 11(9):1559-1566.

7. Smith $A D$, Sumazin $P$, Zhang MQ: Identifying tissue-selective transcription factor binding sites in vertebrate promoters. Proc Natl Acad Sci USA 2005, 102(5):1560-1565.

8. Smith $A D$, Sumazin $P$, Zhang MQ: Tissue-specific regulatory elements in mammalian promoters. Mol Syst Biol 2007, 3:73.

9. Smith $A D$, Sumazin $P$, Xuan Z, Zhang MQ: DNA motifs in human and mouse proximal promoters predict tissue-specific expression. Proc Natl Acad Sci USA 2006, 103(16):6275-6280.

10. Heintzman ND, Hon GC, Hawkins RD, Kheradpour P, Stark A, Harp LF, Ye Z, Lee LK, Stuart RK, Ching CW, et al: Histone modifications at human enhancers reflect global cell-type-specific gene expression. Nature 2009, 459(7243):108-112.

11. Carninci P, Kasukawa T, Katayama S, Gough J, Frith MC, Maeda N, Oyama R, Ravasi T, Lenhard B, Wells C, et al: The transcriptional landscape of the mammalian genome. Science 2005, 309(5740):1559-1563.

12. Carninci P, Sandelin A, Lenhard B, Katayama S, Shimokawa K, Ponjavic J, Semple CA, Taylor MS, Engstrom PG, Frith MC, et al: Genome-wide analysis of mammalian promoter architecture and evolution. Nat Genet 2006, 38(6):626-635

13. Wang Z, Zang C, Rosenfeld JA, Schones DE, Barski A, Cuddapah S, Cui K, Roh TY, Peng W, Zhang MQ, et al: Combinatorial patterns of histone acetylations and methylations in the human genome. Nat Genet 2008, 40(7):897-903.

14. Barski A, Cuddapah S, Cui K, Roh TY, Schones DE, Wang Z, Wei G, Chepelev I, Zhao K: High-resolution profiling of histone methylations in the human genome. Cell 2007, 129(4):823-837.

15. Cui K, Zang C, Roh TY, Schones DE, Childs RW, Peng W, Zhao K: Chromatin signatures in multipotent human hematopoietic stem cells indicate the fate of bivalent genes during differentiation. Cell Stem Cell 2009, 4(1):80-93

16. Karlic R, Chung HR, Lasserre J, Vlahovicek K, Vingron M: Histone modification levels are predictive for gene expression. Proc Natl Acad Sci USA 2010, 107(7):2926-2931.

17. Pekowska A, Benoukraf T, Ferrier P, Spicuglia S: A unique H3K4me2 profile marks tissue-specific gene regulation. Genome Res 2010, 20:1493-1502.

18. Zhao $X Y$, Xuan $Z Y$, Zhang MQ: Boosting with stumps for predicting transcription start sites. Genome biology 2007, 8(2):R17.

19. Wang $X W$, Xuan $Z Y$, Zhao XY, Li YD, Zhang MQ: High-resolution human core-promoter prediction with CoreBoost_HM. Genome Res 2009, 19(2):266-275.

20. Su Al, Wiltshire T, Batalov S, Lapp H, Ching KA, Block D, Zhang J, Soden R, Hayakawa M, Kreiman G, et al: A gene atlas of the mouse and human protein-encoding transcriptomes. Proc Natl Acad Sci USA 2004, 101(16):6062-6067.

21. Bajic VB, Seah SH, Chong A, Zhang G, Koh JL, Brusic V: Dragon Promoter Finder: recognition of vertebrate RNA polymerase II promoters. Bioinformatics 2002, 18(1):198-199.

22. Davuluri RV, Grosse I, Zhang MQ: Computational identification of promoters and first exons in the human genome. Nat Genet 2001, 29(4):412-417.

23. Marson A, Levine SS, Cole MF, Frampton GM, Brambrink T, Johnstone $S$, Guenther MG, Johnston WK, Wernig M, Newman J, et al: Connecting microRNA genes to the core transcriptional regulatory circuitry of embryonic stem cells. Cell 2008, 134(3):521-533.

24. Mikkelsen TS, Ku M, Jaffe DB, Issac B, Lieberman E, Giannoukos G, Alvarez P, Brockman W, Kim TK, Koche RP, et al: Genome-wide maps of chromatin state in pluripotent and lineage-committed cells. Nature 2007, 448(7153):553-560

25. Roh TY, Cuddapah S, Cui K, Zhao K: The genomic landscape of histone modifications in human T cells. Proc Natl Acad Sci USA 2006, 103(43):15782-15787.

26. Saxonov S, Berg P, Brutlag DL: A genome-wide analysis of CpG dinucleotides in the human genome distinguishes two distinct classes of promoters. Proc Natl Acad Sci USA 2006, 103(5):1412-1417.

27. Bajic VB, Tan SL, Suzuki Y, Sugano S: Promoter prediction analysis on the whole human genome. Nat Biotechnol 2004, 22(11):1467-1473.

28. Abeel T, Saeys Y, Bonnet E, Rouze P, Van de Peer Y: Generic eukaryotic core promoter prediction using structural features of DNA. Genome Res 2008, 18(2):310-323.
29. Cenik C, Derti A, Mellor JC, Berriz GF, Roth FP: Genome-wide functional analysis of human 5'untranslated region introns. Genome biol 2010, 11(3): R29.

30. Nogami H, Hoshino R, Ogasawara K, Miyamoto S, Hisano S: Region-specific expression and hormonal regulation of the first exon variants of rat prolactin receptor mRNA in rat brain and anterior pituitary gland. $J$ Neuroendocrinol 2007, 19(8):583-593.

31. Turner JD, Schote AB, Macedo JA, Pelascini LP, Muller CP: Tissue specific glucocorticoid receptor expression, a role for alternative first exon usage? Biochem Pharmacol 2006, 72(11):1529-1537.

32. Roh TY, Wei G, Farrell CM, Zhao K: Genome-wide prediction of conserved and non-conserved enhancers by histone acetylation patterns. Genome Res 2007, 17(1):74-81.

33. Schones DE, Zhao K: Genome-wide approaches to studying chromatin modifications. Nat Rev Genet 2008, 9(3):179-191.

34. Wei G, Wei L, Zhu J, Zang C, Hu-Li J, Yao Z, Cui K, Kanno Y, Roh TY, Watford WT, et al: Global mapping of H3K4me3 and H3K27me3 reveals specificity and plasticity in lineage fate determination of differentiating CD4+ T cells. Immunity 2009, 30(1):155-167.

35. Lorincz MC, Dickerson DR, Schmitt M, Groudine M: Intragenic DNA methylation alters chromatin structure and elongation efficiency in mammalian cells. Nat Struct Mol Biol 2004, 11(11):1068-1075.

36. Birney E, Stamatoyannopoulos JA, Dutta A, Guigo R, Gingeras TR, Margulies EH, Weng Z, Snyder M, Dermitzakis ET, Thurman RE, et al: Identification and analysis of functional elements in $1 \%$ of the human genome by the ENCODE pilot project. Nature 2007, 447(7146):799-816.

37. Valen E, Pascarella G, Chalk A, Maeda N, Kojima M, Kawazu C, Murata M, Nishiyori H, Lazarevic D, Motti D, et al: Genome-wide detection and analysis of hippocampus core promoters using DeepCAGE. Genome Res 2009, 19(2):255-265.

38. Brunner AL, Johnson DS, Kim SW, Valouev A, Reddy TE, Neff NF, Anton E, Medina C, Nguyen L, Chiao E, et al: Distinct DNA methylation patterns characterize differentiated human embryonic stem cells and developing human fetal liver. Genome Res 2009, 19(6):1044-1056.

39. Portela A, Esteller M: Epigenetic modifications and human disease. Nat Biotechnol 2010, 28(10):1057-1068.

40. Maunakea AK, Nagarajan RP, Bilenky M, Ballinger TJ, D'Souza C, Fouse SD, Johnson BE, Hong C, Nielsen C, Zhao Y, et al: Conserved role of intragenic DNA methylation in regulating alternative promoters. Nature 2010, 466(7303):253-257.

41. Heintzman ND, Stuart RK, Hon G, Fu YT, Ching CW, Hawkins RD, Barrera LO, Van Calcar S, Qu CX, Ching KA, et al: Distinct and predictive chromatin signatures of transcriptional promoters and enhancers in the human genome. Nat Genet 2007, 39(3):311-318.

42. Roh TY, Cuddapah S, Zhao K: Active chromatin domains are defined by acetylation islands revealed by genome-wide mapping. Genes Dev 2005, 19(5):542-552

43. Guenther MG, Levine SS, Boyer LA, Jaenisch R, Young RA: A chromatin landmark and transcription initiation at most promoters in human cells. Cell 2007, 130(1):77-88

44. Core L, Waterfall JJ, Lis JT: Nascent RNA sequencing reveals widespread pausing and divergent initiation at human promoters. Science 2008 322(5909):1845-1848.

45. Core $L$, Lis JT: Transcription regulation through promoter-proximal pausing of RNA polymerase II. Science 2008, 319(5871):1791-1792.

46. Seila AC, Calabrese JM, Levine SS, Yeo GW, Rahl PB, Flynn RA, Young RA, Sharp PA: Divergent transcription from active promoters. Science 2008, 322(5909):1849-1851.

47. Bartel DP: MicroRNAs: genomics, biogenesis, mechanism, and function. Cell 2004, 116(2):281-297.

48. Bartel DP: MicroRNAs: target recognition and regulatory functions. Cell 2009, 136(2):215-233

49. Cai $\mathrm{X}$, Hagedorn $\mathrm{CH}$, Cullen BR: Human microRNAs are processed from capped, polyadenylated transcripts that can also function as mRNAs. RNA 2004, 10(12):1957-1966.

50. Lee Y, Kim M, Han J, Yeom KH, Lee S, Baek SH, Kim VN: MicroRNA genes are transcribed by RNA polymerase II. EMBO J 2004, 23(20):4051-4060.

51. Borchert GM, Lanier W, Davidson BL: RNA polymerase III transcribes human microRNAs. Nat Struct Mol Biol 2006, 13(12):1097-1101.

52. Landgraf $P$, Rusu M, Sheridan R, Sewer A, lovino N, Aravin A, Pfeffer S, Rice A, Kamphorst AO, Landthaler $\mathrm{M}$, et al: A mammalian microRNA 
expression atlas based on small RNA library sequencing. Cell 2007, 129(7):1401-1414.

53. Xuan Z, Zhao F, Wang J, Chen G, Zhang MQ: Genome-wide promoter extraction and analysis in human, mouse, and rat. Genome biol 2005, 6(8):R72.

54. Hastie T, Tibshirani R, Friedman J: The elements of satistical learning: Data mining, inference, and prediction. New York: Springer-Verlag; 2000

55. Breiman L, Friedman J, Olshen R, Storne C: Classification and regression trees. Belmont, CA: Wadsworth International Group; 1984

doi:10.1186/1471-2105-12-155

Cite this article as: Zhang and Zhang: Histone modification profiles are predictive for tissue/cell-type specific expression of both protein-coding and microRNA genes. BMC Bioinformatics 2011 12:155.

Submit your next manuscript to BioMed Central and take full advantage of:

- Convenient online submission

- Thorough peer review

- No space constraints or color figure charges

- Immediate publication on acceptance

- Inclusion in PubMed, CAS, Scopus and Google Scholar

- Research which is freely available for redistribution

Submit your manuscript at www.biomedcentral.com/submit 